\title{
$\left.{ }^{177} \mathrm{Lu}\right] \mathrm{Lu}-\mathrm{DOTA}-\mathrm{ZOL}$ bone pain palliation in patients with skeletal metastases from various cancers: efficacy and safety results
}

\author{
Madhav Prasad Yadav ${ }^{1 \dagger}$, Sanjana Ballal ${ }^{1 \dagger}$, Marian Meckel ${ }^{2}$, Frank Roesch ${ }^{2}$ and Chandrasekhar Bal ${ }^{1 *}$ (1)
}

\begin{abstract}
Background: [ $\left.{ }^{177} \mathrm{Lu}\right] \mathrm{Lu}-\mathrm{DOTA}-\mathrm{ZOL}$ has shown promising results from the dosimetry and preclinical aspects, but data on its role in the clinical efficacy are limited. The objective of this study is to evaluate the efficacy and safety of [ $\left.{ }^{177} \mathrm{Lu}\right]$ Lu-DOTA-ZOL as a bone pain palliation agent in patients experiencing pain due to skeletal metastases from various cancers.

Methods: In total, 40 patients experiencing bone pain due to skeletal metastases were enrolled in this study. The

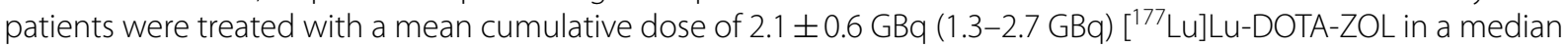
follow-up duration of 10 months (IQR 8-14 months). The primary outcome endpoint was response assessment according to the visual analogue score (VAS). Secondary endpoints included analgesic score (AS), global pain assessment score, Eastern Cooperative Oncology Group Assessment performance status (ECOG), Karnofsky performance status, overall survival, and safety assessment by the National Cancer Institute's Common Toxicity Criteria V5.0.
\end{abstract}

Results: In total, 40 patients (15 males and 25 females) with a mean age of $46.6 \pm 15.08$ years (range 24-78 years) were treated with either $1(N=15)$ or $2(N=25)$ cycles of [ $\left.{ }^{177} \mathrm{Lu}\right] \mathrm{Lu}-\mathrm{DOTA}-Z \mathrm{OLL}$. According to the VAS response assessment criteria, complete, partial, and minimal responses were observed in 11 (27.5\%), 20 (50\%), and 5 patients (12.5\%), respectively with an overall response rate of $90 \%$. Global pain assessment criteria revealed complete, partial, minimal, and no response in $2(5 \%), 25$ (62.5\%), 9 (22.5\%), and 4 (10\%) patients, respectively. Twenty-eight patients died and the estimated median overall survival was 13 months ( $95 \% \mathrm{Cl} 10-14$ months). A significant improvement was observed in the VAS, AS, and ECOG status when compared to baseline. None of the patients experienced grade III/IV haematological, kidney, or hepatotoxicity due to [ ${ }^{177}$ Lu]Lu-DOTA-ZOL therapy.

Conclusion: $\left.{ }^{177} \mathrm{Lu}\right] \mathrm{Lu}-\mathrm{DOTA}-\mathrm{ZOL}$ shows promising results and is an effective radiopharmaceutical in the treatment of bone pain due to skeletal metastases from various cancers.

Keywords: $\left[{ }^{177}\right.$ Lu]Lu-DOTA-ZOL, Pain palliation, Skeletal metastases

*Correspondence: csbal@hotmail.com

${ }^{\dagger}$ Madhav Prasad Yadav and Sanjana Ballal have contributed equally to this work

${ }^{1}$ Department of Nuclear Medicine, Room No: 59-A, Thyroid Clinic, All India Institute of Medical Sciences (AllMS), Ansari Nagar, New Delhi 110029, India

Full list of author information is available at the end of the article

\begin{abstract}
Introduction
Bone is the most common site of metastases in the majority of the solid cancers. Skeletal metastases from prostate and breast cancer account for approximately $80 \%$ of all the bone metastases followed by lung and renal cancers that comprise $20-40 \%$ of all the patients [1]. The typical clinical symptom of skeletal metastases is bone pain. Apart from pain, other skeletal-related events (SREs), albeit to a lesser extent, are swelling, nerve compression,
\end{abstract}

Springer Open

C The Author(s) 2020. Open Access This article is licensed under a Creative Commons Attribution 4.0 International License, which permits use, sharing, adaptation, distribution and reproduction in any medium or format, as long as you give appropriate credit to the original author(s) and the source, provide a link to the Creative Commons licence, and indicate if changes were made. The images or other third party material in this article are included in the article's Creative Commons licence, unless indicated otherwise in a credit line to the material. If material is not included in the article's Creative Commons licence and your intended use is not permitted by statutory regulation or exceeds the permitted use, you will need to obtain permission directly from the copyright holder. To view a copy of this licence, visit http://creativecommons.org/licenses/by/4.0/. 
immobility, or pathological fractures. At the same time, few patients are even asymptomatic, particularly in the early part of the disease with marrow metastases.

Several algorithms have been evolved over the last 3 decades for the management of metastatic bone pain and SREs [2] in which a range of systemic to locoregional therapies are advocated. The most common approaches in clinical practice are chemotherapy, hormonal therapy, bisphosphonates, monoclonal antibody, namely denosumab and analgesics like non-steroidal anti-inflammatory drugs and opioids, molecules signalling growth factors, antidepressants, and endothelin receptor antagonists. Locoregional therapies are offered only for patients with oligo-metastases, namely, external beam radiotherapy (EBRT). Even though the list seems vast, none of them are curative in practice, with a majority of patients limited to palliative care, and involve a multidisciplinary approach.

Palliative external beam radiotherapy is the most effective way of bone pain management. Studies have revealed that $80-90 \%$ of patients receiving EBRT have demonstrated complete or partial pain relief within 10-14 days from initiation of treatment. However, EBRT, as mentioned above, is only limited to the treatment of oligometastases or in a worst-case scenario to hemi-body irradiation [3].

Radionuclide therapy is a systemic form of internal radiotherapy which constitutes an essential option as a routine part of the multidisciplinary treatment approach for decades. Several beta-emitting radiometals have been exploited for this purpose that includes ${ }^{89} \mathrm{Sr}{ }^{186} \mathrm{Re},{ }^{188} \mathrm{Re}$, and ${ }^{153} \mathrm{Sm}$. All of them have been incorporated into boneseeking phosphonates, except ${ }^{89} \mathrm{Sr}$. The ${ }^{89} \mathrm{Sr}$ is a bivalent cation sharing properties similar to calcium. The same is true for ${ }^{223} \mathrm{Ra}$, the most recent radionuclide which has been approved for pain palliation in prostate cancer without visceral metastasis [4], except that it is an alpha emitter. However, the availability and the cost of ${ }^{223} \mathrm{Ra}$ pose paramount restraints for most of the developing world. In this scenario, beta emitters are still affordable and an acceptable option. Owing to the suitable physical properties and decay characteristics of ${ }^{177} \mathrm{Lu}[t 1 / 2=6.73$ days $\mathrm{E} \beta \max =497 \mathrm{keV}, \mathrm{E} \gamma)=113 \mathrm{keV}(6.4 \%), 208 \mathrm{keV}(11 \%)]$, it is now widely used in clinical practice. It is either produced via the ${ }^{176} \mathrm{Lu}(\mathrm{n}, \gamma)[5]$ or the ${ }^{176} \mathrm{Yb}(\mathrm{n}, \gamma)$ pathway [6].

For bone pain palliation it has been labelled with several bisphosphonates such as ethylene diamine tetramethylene phosphonic acid (EDTMP) [7-9], (4-\{[(bis(phosphonomethyl)) carbamoyl]methyl\}-7,10-bi (carboxymethyl)-1,4,7,10-tetraazacyclododec-1-yl) acetic acid (BPAMD) [10-15] Zoledronate (DOTA-ZOL) [16-22], and DOTMP [23, 24]. Zoledronic acid, compared to EDTMP and DOTMP, has significantly higher hydroxyapatite binding and better internalisation by osteoclasts. These superior antiresorptive properties lead to increased apoptosis [25]. DOTA-ZOL can be labelled with both ${ }^{68} \mathrm{Ga}$ and ${ }^{177} \mathrm{Lu}$ to form a theranostic pair [19]. However, only two studies have reported its safety from the dosimetric aspects [26, 27]. Recently, in a pilot study, Nikzad et al. [26] labelled DOTA-zoledronate with ${ }^{177} \mathrm{Lu}$ and have shown promising results and comparable pharmacokinetics to $\left[{ }^{177} \mathrm{Lu}\right] \mathrm{Lu}-\mathrm{EDTMP}$. While dosimetry data revealed a higher absorbed dose for $\left[{ }^{177} \mathrm{Lu}\right]$ Lu-DOTA-ZOL compared to [ ${ }^{177} \mathrm{Lu}$ ]Lu-EDTMP (12.17 vs. $10.02 \mathrm{mSv} / \mathrm{MBq}$ ) in the trabecular bone surface, the absorbed dose to the critical organs and the muscle from $\left[{ }^{177} \mathrm{Lu}\right] \mathrm{Lu}$-DOTA-ZOL was much lower compared to that of $\left[{ }^{177} \mathrm{Lu}\right] \mathrm{Lu}$-EDTMP [27] consistent with the results of the above study. Khawar et al. [21] revealed a similar biodistribution and normal organ absorbed doses of $\left[{ }^{177} \mathrm{Lu}\right] \mathrm{Lu}$-DOTAZOL. However, to the best of our knowledge, investigation on the efficacy and safety of $\left[{ }^{177} \mathrm{Lu}\right] \mathrm{Lu}-\mathrm{DOTA}-\mathrm{ZOL}$ for pain palliation in a clinical setting has not been reported to date. Hence, in the present study, we aim to report the efficacy and toxicity data of the patients, treated with $\left[{ }^{177} \mathrm{Lu}\right] \mathrm{Lu}-\mathrm{DOTA}-\mathrm{ZOL}$, for the bone pain palliation of skeletal metastases from various cancers.

\section{Materials and methods}

The study was conducted at the Department of Nuclear Medicine, AIIMS, New Delhi, India. Skeletal metastases patients suffering from pain were referred from the Pain Clinic, Medical Oncology, and Radiation Oncology departments for $\left[{ }^{177} \mathrm{Lu}\right] \mathrm{Lu}-\mathrm{DOTA}-\mathrm{ZOL}$ pain palliation treatment. This cohort study involved patients who were treated with $\left[{ }^{177} \mathrm{Lu}\right] \mathrm{Lu}-\mathrm{DOTA}-\mathrm{ZOL}$ for pain palliation between January 2017 and February 2020.

\section{Eligibility criteria}

Eligibility criteria for the $\left[{ }^{177} \mathrm{Lu}\right] \mathrm{Lu}-\mathrm{DOTA}-\mathrm{ZOL}$ pain palliation treatment included: histologically confirmed breast, prostate, or lung cancers, progressive pain or pain requiring escalation of analgesics, patients with more than one site of pain corresponding to the avid uptake on $\left[{ }^{68} \mathrm{Ga}\right] \mathrm{Ga}-\mathrm{DOTA}-\mathrm{ZOL} \mathrm{PET} / \mathrm{CT}$ scan, patients with no prior history of radionuclide pain palliation therapy, Eastern Cooperative Oncology Group (ECOG) performance status $\leq 4, K P S \geq 50$, patient on or with history of prior bisphosphonates, patients with haematological, kidney, and liver function parameters within normal limits which included baseline haemoglobin of $<9 \mathrm{~g} / \mathrm{dL}$, platelet counts: $<75,000 / \mu \mathrm{L}$, leukocyte counts: $\geq 4 \times 10^{9} / \mathrm{L}$, serum creatinine: $>1.4 \mathrm{mg} / \mathrm{dL}$, serum bilirubin $>3 \mathrm{mg} \%$, glomerular filtration rate (GFR): $<50 \mathrm{~mL} /$ min per $1.73 \mathrm{~m}^{2}$ body surface area (BSA). Patients with 
skeletal-related events involving pathological fractures and cord compression were not included.

\section{[ ${ }^{177} \mathrm{Lu}$ Lu-DOTA-ZOL synthesis}

The stock solution consisted of $1 \mathrm{mg}$ DOTA-ZOL dissolved in $1 \mathrm{~mL}$ ultrapure water to give a concentration of $60 \mu \mathrm{g} / 60 \mu \mathrm{L}$. The $60 \mu \mathrm{L}$ of DOTA-ZOL was radiolabelled with $\left[{ }^{177} \mathrm{Lu}\right] \mathrm{LuCl}_{3}$ which was obtained from BRIT, India, in sodium ascorbate buffer, $\mathrm{pH} 4$, in $0.01 \mathrm{M}$ supra pure $\mathrm{HCl}$ with a specific activity ranging between 370 and $740 \mathrm{GBq} / \mathrm{mg}$. The radiolabelled solution was heated at $95{ }^{\circ} \mathrm{C}$ for $30 \mathrm{~min}$. Radiochemical quality control was carried out using the instant thin-layer chromatography method with sodium citrate buffer as the solvent.

\section{Treatment protocol and follow-up}

\section{${ }^{177}$ Lu]Lu-DOTA-ZOL infusion}

The patients who fulfilled the eligibility criteria were administered with a fixed dose of $1295 \mathrm{MBq}(35 \mathrm{mCi}$ ) $\left[{ }^{177} \mathrm{Lu}\right] \mathrm{Lu}-\mathrm{DOTA}-\mathrm{ZOL}$. The fixed activity of $1295 \mathrm{MBq}$ of $\left[{ }^{177} \mathrm{Lu}\right] \mathrm{Lu}-\mathrm{DOTA}-\mathrm{ZOL}$ was extrapolated from our previous $\left[{ }^{177} \mathrm{Lu}\right] \mathrm{Lu}$-EDTMP phase II data [8]. The infusion involved a dilution of $\left[{ }^{177} \mathrm{Lu}\right] \mathrm{Lu}-\mathrm{DOTA}-\mathrm{ZOL}$ in $10 \mathrm{~mL}$ normal saline $(0.9 \%)$, which was administered intravenously over $5 \mathrm{~min}$, with subsequent flushing of $20 \mathrm{~mL}$ normal saline. The entire process was performed on an in-patient basis, and patients were discharged in a few hours of observation if they do not show any adverse reaction to $\left[{ }^{177} \mathrm{Lu}\right] \mathrm{Lu}-\mathrm{DOTA}-\mathrm{ZOL}$. Figure 1 shows biodistribution and uptake of $\left[{ }^{177} \mathrm{Lu}\right] \mathrm{Lu}-\mathrm{DOTA}-\mathrm{ZOL}$ therapy in a patient with skeletal metastases from prostate cancer, while Fig. 2 gives similar data of $\left[{ }^{177} \mathrm{Lu}\right] \mathrm{Lu}-\mathrm{DOTA}-\mathrm{ZOL}$ therapy in a patient with skeletal metastases from breast cancer.

\section{Follow-up}

The treatment was repeated if necessary at 3-monthly intervals. Post- $\left[{ }^{177} \mathrm{Lu}\right] \mathrm{Lu}-\mathrm{DOTA}-\mathrm{ZOL}$ administration, patients were assessed at $2,4,8$, and 12 weeks. Patients were assessed for laboratory parameters, and the adverse events were recorded according to the National Cancer Institute for Common Toxicity Criteria version (CTC) 5.0 [28]. The visual analogue score (VAS) [29], global pain assessment score, analgesic score (AS), Karnofsky performance status (KPS) [30, 31], and assessment of pain relief were recorded in the patient case files on each visit. Patients were instructed to maintain a diary and document the pain relief parameters such as initiation of pain relief, duration of pain relief, time of increase and/or

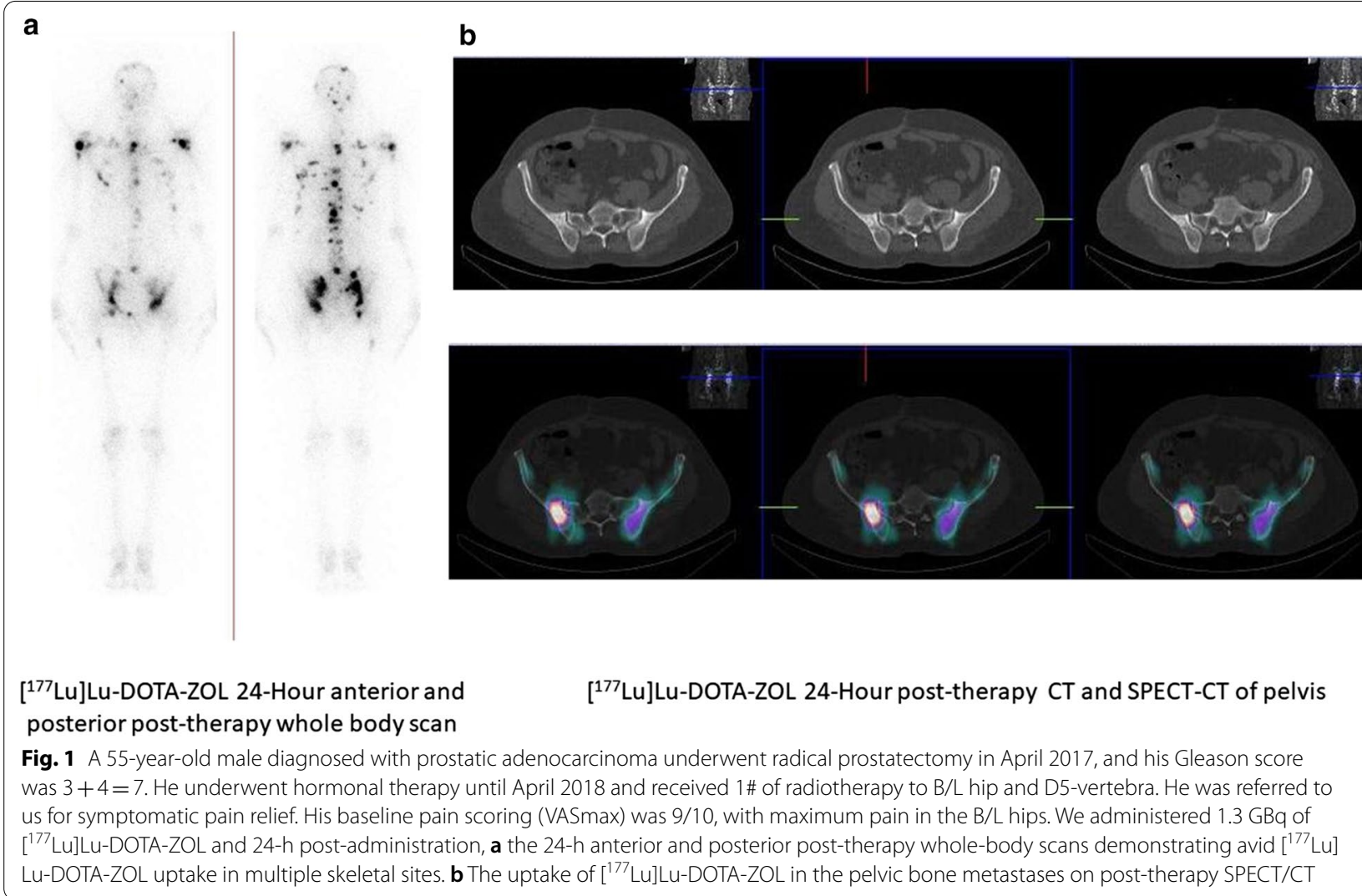


a

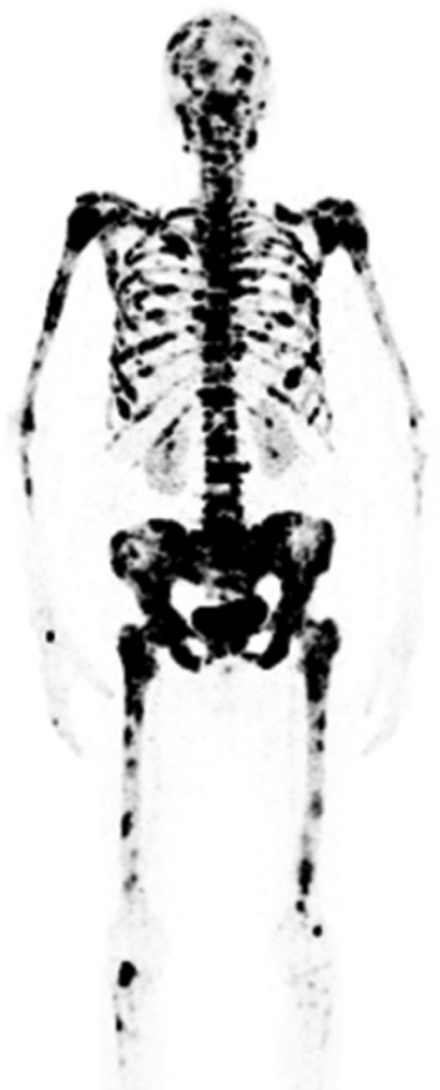

b
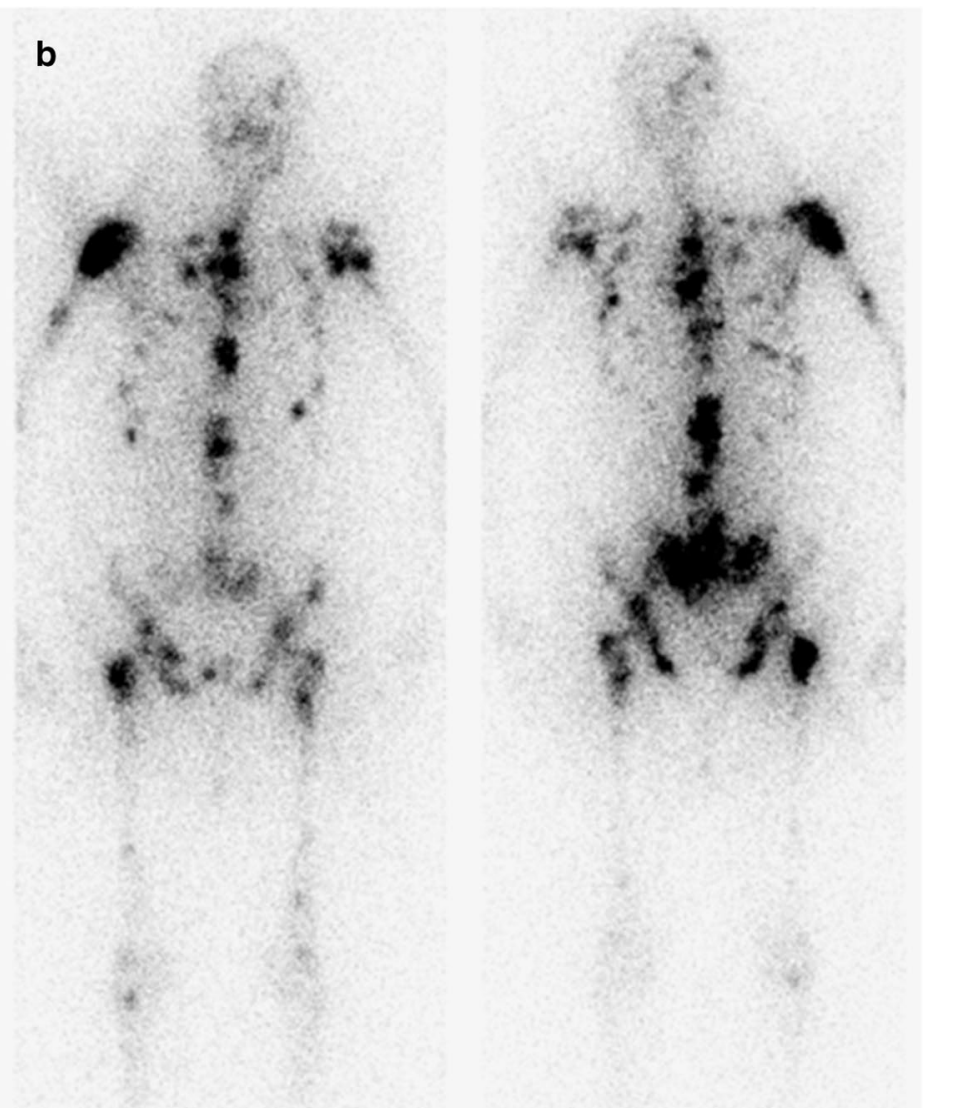

$\left[{ }^{68} \mathrm{Ga}\right] \mathrm{Ga}-\mathrm{DOTA}-Z O L$ MIP

\section{[177Lu]Lu-DOTA-ZOL 24-Hour anterior and posterior post-therapy whole body scan}

Fig. 2 A 45-year-old female, diagnosed with triple-negative breast cancer was on opioid medications, but did not experience any relief in pain. Her baseline pain scoring (VASmax) was 8/10. Baseline $\left[{ }^{68} \mathrm{Ga}\right] \mathrm{Ga}$-DOTA-ZOL PET/CT (a) was conducted followed by administration of $1.3 \mathrm{GBq}$ of $\left[{ }^{177} \mathrm{Lu}\right] \mathrm{Lu}-\mathrm{DOTA}-Z \mathrm{OL}$. Twenty-four-hour post-administration, post-therapy 24-h anterior and posterior whole body scan (b) demonstrate avid [ $\left.{ }^{177} \mathrm{Lu}\right]$ Lu-DOTA-ZOL uptake in multiple skeletal sites

recurrence of pain, decrease or increase in the consumption of pain killers.

\section{Treatment response assessment Primary outcome endpoint}

The primary outcome endpoint was response assessment by VAS. According to this criteria, the complete response $(\mathrm{CR})$, partial response (PR), minimal response(MR), and no response (NR) were categorised as $>70 \%$ reduction,
$40-70 \%$ reduction, $20-40 \%$ reduction, and $<20 \%$ decrease in VAS or increase in pain, respectively [29].

\section{Secondary outcome endpoints}

Other clinical response assessment parameters involved analgesic score (AS), Karnofsky performance status (KPS), Eastern Cooperative Oncology Group (ECOG) performance status, global pain assessment, adverse event profile, and overall survival. 
Analgesic scoring was conducted as per the Urological Group of the European Organization of Research and Treatment of Cancer (EORTC, Protocol 30921). As per EORTC protocol, the analgesic score is the product of two five-point scales (the type of analgesic and the frequency of its administration). A decline in the analgesic score was documented as a response to treatment.

Additionally, global pain response assessment was analysed according to the criteria adopted by Thapa et al. [32] that considered changes in both VAS and analgesic scores (rather than a single parameter). According to the criteria, the present study design considered post-therapy changes in both VAS and analgesic scores on a sliding scale. The global pain assessment criteria are accordingly complete $(75 \%$ decrease in analgesic score with change in pain score), partial (50-75\% decrease in analgesic score with a change in pain score), minimal (25-50\% decrease in analgesic score with a change in pain score), or none (no change in pain score or, $25 \%$ decrease in the analgesic score). The KPS was scaled from 100 to 0 . The ECOG status ranged from 0 to 5 . All adverse events were assessed as per the National Cancer Institute's Common Toxicity Criteria (NCI-CTC) version 5.0. The overall survival (OS) was defined as the time from the initiation of $\left[{ }^{177} \mathrm{Lu}\right] \mathrm{Lu}-\mathrm{DOTA}-\mathrm{ZOL}$ treatment to the time of death. The death could be attributed to any cause or the last telephonic contact.

\section{Statistical analysis}

The normality of the data was examined by the D'Agostino-Pearson test. The data were presented as mean standard deviation (SD), median, and/or interquartile range (IQR). Unpaired samples $t$ test (parametric test) or Mann-Whitney $U$ test (nonparametric test) was performed for two independent patient groups. The paired $t$ test (parametric test) or Wilcoxon signed-rank test (nonparametric test) was executed to compare parameters at pre- and post-treatment time points. Kaplan-Meier curves analysis was conducted to calculate the overall survival. MedCalc software was used for statistical analyses. $P$ values $\leq 0.05$ were considered significant.

\section{Results}

\section{Patients}

Forty documented skeletal metastases patients including 15 males and 25 females with a mean age of $46.6 \pm 15.08$ years (range $24-78$ years) were enrolled and treated with $\left[{ }^{177} \mathrm{Lu}\right] \mathrm{Lu}$-DOTA-ZOL for bone pain palliation therapy. The patients were treated between January 2017 and February 2020 with the median follow-up duration of 10 (IQR 8-14) months.

The baseline demographic profile of the patients, tumour characteristics, previous and ongoing cancer-related treatments, and the analgesics consumed are outlined in Tables 1 and 2. Among the patients treated, breast cancer $(23 / 40,57.5 \%)$ accounted for the maximum number of cases followed by prostate cancer (11/40, 27.5\%). The remaining 6 patients had lung cancer (Table 1). Except eight patients with prostate cancer who were on concomitant hormonal therapy, no other patients were on any anti-cancer treatment during the treatment. While $15(37.5 \%)$ patients were on morphine medications at the baseline, the remaining patients $(62.5 \%)$ were either on atypical opioids, non-morphine opioids, or other NSAIDs. Before being referred to our department for bone pain palliation, all the patients had undergone a minimum of two lines of prior treatment.

\section{Treatment cycles and efficacy assessment}

The cumulative activity administered was $2.1 \pm 0.6 \mathrm{GBq}$ (range 1.3-2.7 GBq) $(56.8 \pm 17 \mathrm{mCi}$; range $35-70 \mathrm{mCi})$. A total of 65 cycles of $\left[{ }^{177} \mathrm{Lu}\right] \mathrm{Lu}-\mathrm{DOTA}-\mathrm{ZOL}$ were administered in 40 patients, among whom, 25 patients received two cycles each and the remaining 15 patients received only 1 cycle. Flair phenomenon was noted in 7/40 (17.5\%) of patients within $2-3$ days of $\left[{ }^{177} \mathrm{Lu}\right] \mathrm{Lu}-\mathrm{DOTA}-\mathrm{ZOL}$ treatment. These patients complained of persistent pain even on strong analgesics; however, it was transient and

Table 1 Patient clinical characteristics

\begin{tabular}{ll}
\hline Variables & Number (\%) \\
\hline $\begin{array}{l}\text { Age in years (mean } \pm \text { SD, range) } \\
\text { Gender }\end{array}$ & $46.6 \pm 15.08(24-78)$ \\
Male & $15(37.5 \%)$ \\
Female & $25(62.5 \%)$ \\
Primary disease & \\
Prostate & $11(27.5 \%)$ \\
Breast & $23(57.5 \%)$ \\
Lung & $6(15 \%)$ \\
Baseline VAS pre-therapy (median, IQR) & $9(8-10)$ \\
Baseline KPS pre-therapy (median, IQR) & $60(50-70)$ \\
Baseline analgesic score (median) & $6(6-8)$ \\
The extent of skeletal metastases & \\
$<6$ & $2(5 \%)$ \\
$6-20$ & $17(42.5 \%)$ \\
$>20$ & $7(17.5 \%)$ \\
Diffuse/superscan & $14(35 \%)$ \\
ECOG performance status & $12(30 \%)$ \\
2 & $15(37.5 \%)$ \\
3 & $13(32.5 \%)$ \\
4 & $2.1 \pm 0.6 \mathrm{GBq}(1.3-2.7 \mathrm{GBq})$ \\
Mean cumulative activity (GBq) &
\end{tabular}

IQR inter-quartile range, VAS visual analogue score, KPS Karnofsky performance status, ECOG Eastern Cooperative Oncology Group, GBq gigabecquerel 
Table 2 Detailed clinical history of patients

\begin{tabular}{|c|c|c|c|c|c|c|}
\hline Patient & Age/gender & Type of cancer & Previous therapy & Ongoing treatment & Analgesic score (type) & $\begin{array}{l}\text { Analgesic } \\
\text { score } \\
\text { (quantity) }\end{array}$ \\
\hline 1 & $51 / \mathrm{M}$ & $\mathrm{mCRPC}$ & $\begin{array}{l}\text { First- and second-gener- } \\
\text { ation anti-androgens, } \\
\text { chemotherapy }\end{array}$ & $\begin{array}{l}\text { Androgen synthesis } \\
\text { inhibitor }\end{array}$ & Morphine & 2 \\
\hline 2 & $25 / F$ & $\begin{array}{l}\text { Non-small cell right lung } \\
\text { cancer }\end{array}$ & $\begin{array}{l}\text { Lobectomy, chemother- } \\
\text { apy, radiotherapy }\end{array}$ & - & Morphine & 2 \\
\hline 3 & $60 / M$ & Right breast cancer & $\begin{array}{l}\text { Chemotherapy, radio- } \\
\text { therapy }\end{array}$ & - & Morphine & 2 \\
\hline 4 & $44 / F$ & $\mathrm{~B} / \mathrm{L}$ breast cancer & $\begin{array}{l}\text { B/L breast mastectomy, } \\
\text { chemotherapy, radio- } \\
\text { therapy, monoclonal } \\
\text { antibody therapy }\end{array}$ & - & Morphine & 2 \\
\hline 5 & $61 / M$ & Right breast cancer & $\begin{array}{l}\text { Hormonal therapy, chem- } \\
\text { otherapy, radiotherapy }\end{array}$ & - & $\begin{array}{l}\text { Atypical opioids and non- } \\
\text { morphine opioids }\end{array}$ & 3 \\
\hline 6 & $70 / \mathrm{M}$ & $\mathrm{mCRPC}$ & $\begin{array}{l}\text { Medical castration, first- } \\
\text { generation anti-andro- } \\
\text { gen therapy, chemo- } \\
\text { therapy, radiotherapy }\end{array}$ & $\begin{array}{l}\text { Second-generation anti- } \\
\text { androgens }\end{array}$ & Morphine & 4 \\
\hline 7 & $63 / M$ & mCRPC & $\begin{array}{l}\text { B/L orchidectomy, first- } \\
\text { generation anti-andro- } \\
\text { gens, chemotherapy, } \\
\text { radiotherapy }\end{array}$ & - & $\begin{array}{l}\text { Atypical opioids and non- } \\
\text { morphine opioids }\end{array}$ & 2 \\
\hline 8 & $60 / F$ & Small-cell lung cancer & $\begin{array}{l}\text { Chemotherapy, radio- } \\
\text { therapy }\end{array}$ & - & $\begin{array}{l}\text { Atypical opioids and non- } \\
\text { morphine opioids }\end{array}$ & 3 \\
\hline 9 & $44 / F$ & Right breast cancer & $\begin{array}{l}\text { Right breast mastectomy, } \\
\text { hormonal therapy, } \\
\text { chemotherapy, radio- } \\
\text { therapy }\end{array}$ & - & $\begin{array}{l}\text { Atypical opioids and non- } \\
\text { morphine opioids }\end{array}$ & 2 \\
\hline 10 & $65 / F$ & Left breast cancer & $\begin{array}{l}\text { Hormonal therapy, } \\
\text { chemotherapy, }\end{array}$ & - & $\begin{array}{l}\text { Atypical opioids and non- } \\
\text { morphine opioids }\end{array}$ & 2 \\
\hline 11 & $33 / F$ & Right breast cancer & $\begin{array}{l}\text { Chemotherapy, radio- } \\
\text { therapy }\end{array}$ & - & Morphine & 2 \\
\hline 12 & $74 / M$ & mCRPC & $\begin{array}{l}\text { Radical Prostatectomy, B/L } \\
\text { orchidectomy, first-gen- } \\
\text { eration anti-androgens, } \\
\text { chemotherapy, }\end{array}$ & - & $\begin{array}{l}\text { Atypical opioids and non- } \\
\text { morphine opioids }\end{array}$ & 2 \\
\hline 13 & $45 / F$ & Left breast cancer & $\begin{array}{l}\text { Left breast modified radi- } \\
\text { cal mastectomy, hormo- } \\
\text { nal therapy, chemother- } \\
\text { apy, radiotherapy, }\end{array}$ & - & $\begin{array}{l}\text { Atypical opioids and non- } \\
\text { morphine opioids }\end{array}$ & 3 \\
\hline 14 & $27 / F$ & $\mathrm{~B} / \mathrm{L}$ breast cancer & $\begin{array}{l}\text { Hormonal therapy, B/L } \\
\text { mastectomy, chemo- } \\
\text { therapy, radiotherapy, }\end{array}$ & - & Morphine & 2 \\
\hline 15 & $47 / F$ & Left breast cancer & $\begin{array}{l}\text { Hormonal therapy, chem- } \\
\text { otherapy, radiotherapy, } \\
\text { antibody therapy }\end{array}$ & - & Other NSAIDs & 1 \\
\hline 16 & $27 / F$ & Left breast cancer & $\begin{array}{l}\text { chemotherapy, radio- } \\
\text { therapy, }\end{array}$ & - & Other NSAIDs & 2 \\
\hline 17 & $55 / M$ & mCRPC & $\begin{array}{l}\text { B/L orchidectomy, first- } \\
\text { and second-generation } \\
\text { anti-androgens, chemo- } \\
\text { therapy, radiotherapy }\end{array}$ & $\begin{array}{l}\text { Androgen synthesis } \\
\text { inhibitors }\end{array}$ & Other NSAIDs & 3 \\
\hline 18 & $44 / F$ & Left breast cancer & $\begin{array}{l}\text { Left modified radical mas- } \\
\text { tectomy, chemotherapy, } \\
\text { radiotherapy }\end{array}$ & - & Morphine & 2 \\
\hline 19 & $58 / F$ & Right breast cancer & $\begin{array}{l}\text { Rt breast mastectomy } \\
\text { chemotherapy, radio- } \\
\text { therapy }\end{array}$ & - & $\begin{array}{l}\text { Atypical opioids and non- } \\
\text { morphine opioids }\end{array}$ & 4 \\
\hline
\end{tabular}


Table 2 (continued)

\section{Patient Age/gender Type of cancer}

Previous therapy
Analgesic score (type) Analgesic score

(quantity)

\begin{tabular}{|c|c|c|c|c|c|c|}
\hline 20 & $24 / M$ & $\begin{array}{l}\text { Squamous cell cancer of } \\
\text { lung }\end{array}$ & Surgery, chemotherapy & - & Morphine & 2 \\
\hline 21 & $55 / F$ & Left breast cancer & $\begin{array}{l}\text { Surgery, chemotherapy, } \\
\text { radiotherapy }\end{array}$ & - & $\begin{array}{l}\text { Atypical opioids and non- } \\
\text { morphine opioids }\end{array}$ & 2 \\
\hline 22 & $55 / F$ & $\begin{array}{l}\text { Squamous cell cancer of } \\
\text { lung }\end{array}$ & $\begin{array}{l}\text { Chemotherapy, radio- } \\
\text { therapy }\end{array}$ & - & $\begin{array}{l}\text { Atypical opioids and non- } \\
\text { morphine opioids }\end{array}$ & 2 \\
\hline 23 & $54 / F$ & Right breast cancer & $\begin{array}{l}\text { Chemotherapy, radio- } \\
\text { therapy }\end{array}$ & - & $\begin{array}{l}\text { Atypical opioids and non- } \\
\text { morphine opioids }\end{array}$ & 3 \\
\hline 24 & $40 / F$ & Right breast cancer & $\begin{array}{l}\text { Chemotherapy, radio- } \\
\text { therapy }\end{array}$ & - & Morphine & 2 \\
\hline 25 & $54 / M$ & mCRPC & $\begin{array}{c}\text { Medical castration, Chem- } \\
\text { otherapy, radiotherapy }\end{array}$ & $\begin{array}{l}\text { Androgen-synthesis } \\
\text { inhibitors }\end{array}$ & Morphine & 2 \\
\hline 26 & $63 / F$ & $\mathrm{~B} / \mathrm{L}$ breast cancer & $\begin{array}{l}\text { Bilateral mastectomy, } \\
\text { chemotherapy, radio- } \\
\text { therapy }\end{array}$ & - & $\begin{array}{l}\text { Atypical opioids and non- } \\
\text { morphine opioids }\end{array}$ & 2 \\
\hline 27 & $47 / F$ & Right breast cancer & $\begin{array}{l}\text { Right breast mastectomy, } \\
\text { hormonal therapy, } \\
\text { chemotherapy, radio- } \\
\text { therapy }\end{array}$ & - & Morphine & 2 \\
\hline 28 & $50 / \mathrm{M}$ & Right breast cancer & $\begin{array}{l}\text { Right modified radical } \\
\text { mastectomy, chemo- } \\
\text { therapy, radiotherapy }\end{array}$ & - & Morphine & 2 \\
\hline 29 & $40 / F$ & $\mathrm{~B} / \mathrm{L}$ breast cancer & $\begin{array}{c}\text { Hormonal therapy, chem- } \\
\text { otherapy, radiotherapy }\end{array}$ & - & Morphine & 2 \\
\hline 30 & $75 / M$ & $\mathrm{mCRPC}$ & $\begin{array}{l}\text { B/L orchidectomy, } \\
\text { chemotherapy, first- and } \\
\text { second-generation anti- } \\
\text { androgens, radiotherapy }\end{array}$ & None & $\begin{array}{l}\text { Atypical opioids and non- } \\
\text { morphine opioids }\end{array}$ & 2 \\
\hline 31 & $67 / M$ & $\mathrm{mCRPC}$ & $\begin{array}{l}\text { B/L orchidectomy, } \\
\text { first-generation anti- } \\
\text { androgens } \\
\text { Chemotherapy, radio- } \\
\text { therapy, }\end{array}$ & $\begin{array}{l}\text { Second-generation anti- } \\
\text { androgens }\end{array}$ & $\begin{array}{l}\text { Atypical opioids and non- } \\
\text { morphine opioids }\end{array}$ & 2 \\
\hline 32 & $78 / \mathrm{M}$ & $\mathrm{mCRPC}$ & $\begin{array}{l}\text { B/L orchidectomy, chemo- } \\
\text { therapy }\end{array}$ & $\begin{array}{l}\text { Androgen synthesis } \\
\text { inhibitor }\end{array}$ & $\begin{array}{l}\text { Atypical opioids and non- } \\
\text { morphine opioids }\end{array}$ & 2 \\
\hline 33 & $48 / F$ & $\mathrm{~B} / \mathrm{L}$ breast cancer & $\begin{array}{l}\text { Chemotherapy, radio- } \\
\text { therapy }\end{array}$ & - & $\begin{array}{l}\text { Atypical opioids and non- } \\
\text { morphine opioids }\end{array}$ & 2 \\
\hline 34 & 40 & Right breast cancer & $\begin{array}{l}\text { Right modified radical } \\
\text { mastectomy, chemo- } \\
\text { therapy, radiotherapy }\end{array}$ & - & $\begin{array}{l}\text { Atypical opioids and non- } \\
\text { morphine opioids }\end{array}$ & 2 \\
\hline 35 & $70 / \mathrm{M}$ & $\mathrm{mCRPC}$ & $\begin{array}{c}\text { Medical castration, chem- } \\
\text { otherapy, radiotherapy }\end{array}$ & $\begin{array}{l}\text { Androgen-synthesis } \\
\text { inhibitors }\end{array}$ & Morphine & 2 \\
\hline 36 & $43 / M$ & $\begin{array}{l}\text { Non-small cell lung cancer } \\
\text { of the right lung }\end{array}$ & $\begin{array}{l}\text { Chemotherapy, radio- } \\
\text { therapy }\end{array}$ & - & $\begin{array}{l}\text { Atypical opioids and non- } \\
\text { morphine opioids }\end{array}$ & 2 \\
\hline 37 & $32 / F$ & Right breast cancer & $\begin{array}{l}\text { Right breast mastectomy } \\
\text { chemotherapy, radio- } \\
\text { therapy }\end{array}$ & - & $\begin{array}{l}\text { Atypical opioids and non- } \\
\text { morphine opioids }\end{array}$ & 2 \\
\hline 38 & $67 / F$ & Right breast cancer & $\begin{array}{l}\text { Right modified radical } \\
\text { mastectomy, hormonal } \\
\text { therapy chemotherapy, } \\
\text { radiotherapy }\end{array}$ & - & $\begin{array}{l}\text { Atypical opioids and non- } \\
\text { morphine opioids }\end{array}$ & 2 \\
\hline 39 & $30 / F$ & Small cell cancer of lung & $\begin{array}{l}\text { Chemotherapy, radio- } \\
\text { therapy }\end{array}$ & - & $\begin{array}{l}\text { Atypical opioids and non- } \\
\text { morphine opioids }\end{array}$ & 3 \\
\hline 40 & $76 / \mathrm{M}$ & $\mathrm{mCRPC}$ & $\begin{array}{l}\text { B/L orchidectomy, first- } \\
\text { generation anti-andro- } \\
\text { gens, chemotherapy, } \\
\text { radiotherapy }\end{array}$ & $\begin{array}{l}\text { Second-generation anti- } \\
\text { androgens }\end{array}$ & $\begin{array}{l}\text { Atypical opioids and non- } \\
\text { morphine opioids }\end{array}$ & 2 \\
\hline
\end{tabular}


Table 2 (continued)

$B / L$ bilateral, $m C R P C$ metastatic castration-resistant prostate cancer

Table 3 Response and number of [ $\left.{ }^{177} \mathrm{Lu}\right] \mathrm{Lu}-\mathrm{DOTA}-\mathrm{ZOL}$ treatment cycles administered according to the extent of disease

\begin{tabular}{|c|c|c|c|c|c|}
\hline $\begin{array}{l}\text { Extent } \\
\text { of skeletal } \\
\text { metastases }\end{array}$ & $\begin{array}{l}\text { Number } \\
\text { of patients } \\
\text { (\%) }\end{array}$ & $\begin{array}{l}\text { Response according } \\
\text { to VAS assessment } \\
\text { criteria (number } \\
\text { of patients) }\end{array}$ & $\begin{array}{l}\text { Number of cycles } \\
\text { administered }\end{array}$ & $\begin{array}{l}\text { Response according } \\
\text { to global pain } \\
\text { assessment criteria } \\
\text { (number of patients) }\end{array}$ & $\begin{array}{l}\text { Number of cycles } \\
\text { administered }\end{array}$ \\
\hline \multirow[t]{2}{*}{$<6$} & $2(5 \%)$ & CR-1 & $2 \mathrm{C}$ & PR-2 & 1st patient-1C \\
\hline & & PR-1 & $1 C$ & & 2nd patient-2C \\
\hline \multirow[t]{4}{*}{$6-20$} & $17(42.5 \%)$ & CR-5 & 4 patients- 2 C, 1 patient- $1 C$ & CR-1 & $2 C$ \\
\hline & & PR-9 & $\begin{array}{l}5 \text { patients-2C, } 4 \text { patients- } \\
1 \mathrm{C}\end{array}$ & PR-11 & 8 patients- 2 C, 3 patients- $1 \mathrm{C}$ \\
\hline & & MR-1 & $2 C$ & MR-3 & 2 patients- $1 C, 1$ patient- $2 C$ \\
\hline & & NR-2 & Both patients-1C & NR-2 & Both patients-1C \\
\hline \multirow[t]{4}{*}{$>20$} & $7(17.5 \%)$ & CR-2 & 2 patients, $2 \mathrm{C}$ & CR-1 & $2 \mathrm{C}$ \\
\hline & & PR-4 & 3 patients- 2 C, 1 patient- $1 C$ & PR-4 & 3 patients- $2 C, 1$ patient- $1 C$ \\
\hline & & MR-0 & 0 & MR-1 & $2 \mathrm{C}$ \\
\hline & & NR-1 & $1 C$ & NR-1 & $1 C$ \\
\hline \multirow[t]{4}{*}{ Superscan/diffuse } & $14(35 \%)$ & CR-3 & All patients-2C & CR-0 & - \\
\hline & & PR-6 & 5 patients- 2 C, 1 patient- $1 \mathrm{C}$ & PR-8 & 6 patients- 2 C, 2 patients- $1 C$ \\
\hline & & MR-4 & 1 patient- 2 C, 3 patients- $1 \mathrm{C}$ & MR-5 & 3 patients-2C, 2 patients- $1 \mathrm{C}$ \\
\hline & & NR-1 & 1 patient $1 C$ & NR-1 & $1 C$ \\
\hline
\end{tabular}

VAS visual analogue score, $C R$ complete response, $P R$ partial response, $M R$ minimal response, $N R$ no response, $C$ cycles

reduced in 7-10-day post-treatment. According to the VAS response criteria, complete response was demonstrated in $27.5 \%(11 / 40)$, partial response in 50\% (20/40), the minimal response in $12.5 \%$ (5/40), and no response in $10 \%(4 / 40)$ of patients with an overall response rate (ORR) of $90 \%$. Similarly, as per the global pain assessment response criteria, pain relief with $\left[{ }^{177} \mathrm{Lu}\right] \mathrm{Lu}-\mathrm{DOTA}-$ ZOL was 90\%: 5\% (2/40) CR, 62.5\% (25/40) PR, and $22.5 \%(9 / 40) \mathrm{MR}$, respectively. The detailed classification of relation between, the extent of skeletal metastases, number of cycles administered, and responses according to both VAS criteria and global pain assessment criteria are explained in Table 3. Interestingly, Among 14 patients with superscan or diffuse involvement of skeletal metastases, only one patient did not respond to treatment. A similar pattern of responses was observed in patients with $>20$ skeletal metastases (Table 3). Nine patients who attained minimal response after the first cycle were re-challenged with the second cycle of $\left[{ }^{177} \mathrm{Lu}\right] \mathrm{Lu}$-DOTAZOL, but found very minimal improvement despite the second cycle and remained in the minimal response category. Among the 15 patients who received only one cycle of $\left[{ }^{177} \mathrm{Lu}\right] \mathrm{Lu}-\mathrm{DOTA}-\mathrm{ZOL}$ treatment, according to VAS criteria, 4 did not respond to treatment, 3 experienced only minimal response, and 8 patients responded well but did not consent for the 2nd cycle of treatment (Table 3).
Table 4 Comparison of pre- and post-treatment analgesic scores stratified according to VAS response criteria

\begin{tabular}{|c|c|c|c|c|}
\hline $\begin{array}{l}\text { Response } \\
\text { (VAS } \\
\text { criteria) }\end{array}$ & $\begin{array}{l}\text { Number } \\
\text { of patients } \\
\text { (N) }\end{array}$ & $\begin{array}{l}\text { Baseline } \\
\text { AS (median, } \\
\text { IQR) }\end{array}$ & $\begin{array}{l}\text { Post-treatment } \\
\text { AS (median, } \\
\text { IQR) }\end{array}$ & $P$ value \\
\hline$C R$ & 11 & $6(6-8)$ & $2(1.2-2.7)$ & 0.001 \\
\hline$P R$ & 20 & $7(6-8)$ & $3(3-3.5)$ & $<0.0001$ \\
\hline MR & 5 & $8(6-8.25)$ & $4.8(4-6)$ & 0.006 \\
\hline NR & 4 & $8.5(8-12.5)$ & $8(8-12)$ & 0.918 \\
\hline
\end{tabular}

All the values are mentioned as median and inter-quartile range (IQR)

VAS visual analogue score, $N$ number of patients, $I Q R$ interquartile range, $C R$ complete response, $P R$ partial response, $M R$ minimal response, $N R$ no response

In the post-treatment period, there was a significant decrease in VAS [(pre-therapy: 9 (IQR 8-10) vs. posttherapy: 4 (IQR 3-5), $P<0.0001)$. Similarly, a remarkable improvement noted in the KPS [(pre-therapy: 60 (IQR 50-70) vs. post-therapy: 80 (IQR 60-80), $P<0.0001$ ), and the ECOG performance status [(pre-therapy: 3 (IQR $2-4)$ vs. post-therapy: 2 (IQR $2-3), P 0.0013)$ post- $\left[{ }^{177} \mathrm{Lu}\right]$ Lu-DOTA-ZOL therapy. While there was a significant reduction in the analgesic score pre- and post-treatment with $\left[{ }^{177} \mathrm{Lu}\right] \mathrm{Lu}-\mathrm{DOTA}-\mathrm{ZOL}$ in the $\mathrm{CR}, \mathrm{PR}$, and the MR categories, the same was not found in the NR category (Table 4). 
Table 5 Response assessment according to the type of cancer

\begin{tabular}{llll}
\hline $\begin{array}{l}\text { Response } \\
\text { criteria }\end{array}$ & $\begin{array}{l}\text { Breast cancer } \\
\boldsymbol{N = 2 3}\end{array}$ & $\begin{array}{l}\text { Prostate cancer } \\
\boldsymbol{N = 1 1}\end{array}$ & $\begin{array}{l}\text { Lung } \\
\text { cancer } \\
\boldsymbol{N}=\mathbf{6}\end{array}$ \\
\hline CR & 9 & 2 & 0 \\
PR & 11 & 7 & 2 \\
$M R$ & 3 & 0 & 2 \\
NR & 0 & 2 & 2 \\
\hline
\end{tabular}

$N$ number of patients, $C R$ complete response, $P R$ partial response, $M R$ minimal response, $N R$ no response

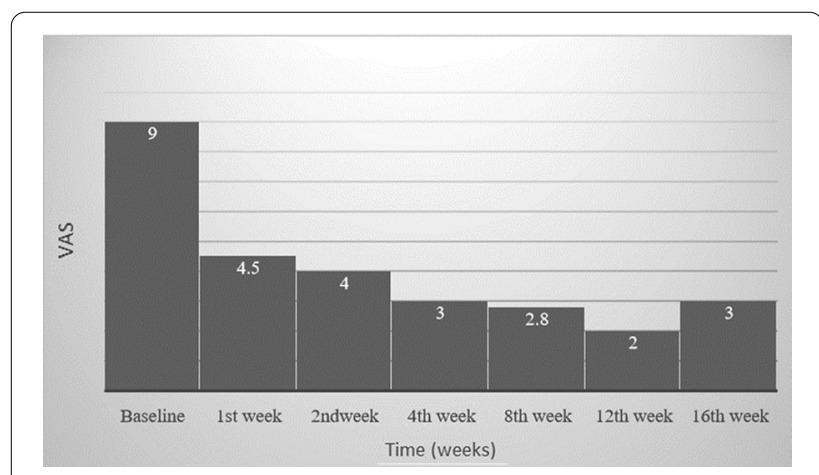

Fig. 3 Plot showing the temporal relationship of the median value of visual analogue score (VAS) of patients from baseline up to 16 weeks of post-[ $\left.{ }^{177} \mathrm{Lu}\right]$ Lu-DOTA-ZOL therapy

Response according to the type of cancer revealed 100\% $(23 / 23), 82 \%(9 / 11)$, and $66.6 \%(4 / 6)$ ORR in patients with breast, prostate, and lung cancer, respectively (Table 5).
The median time for the initiation of pain relief was $\leq 7$ days (IQR 6-9 days) (Fig. 3). The median time of sustained response after the last cycle of the $\left[{ }^{177} \mathrm{Lu}\right]$ Lu-DOTA-ZOL is 3 months (IQR 2-4 months). Only one patient observed the most prolonged duration of sustained response, which was 10 months.

\section{Survival analysis}

Among the entire series, 28 patients died during the follow-up. The median survival from treatment was 13 months (95\% CI 10-14 months), with a 1-year survival probability of $55.4 \%$ (Fig. 4a). On subgroup analysis, in patients with $C R, P R, M R$, and NR, the median overall survival was $13,13,8$, and 5 months, respectively (Fig. 4b). Sub-categorical analysis based on the type of cancer revealed patients with breast and prostate cancer to depict a similar median overall survival duration of 13 months, while patients with lung cancer demonstrated a median overall survival of 10 months. However, the logrank test did not prove significant ( $P$ 0.1431) (Fig. 5).

\section{Toxicity}

The laboratory parameters were tested and analysed for toxicity post-treatment (Table 6). Haematological serious adverse events (SAE) [grade III/IV toxicity] were not observed in any patient despite completing two cycles of $\left[{ }^{177} \mathrm{Lu}\right] \mathrm{Lu}-\mathrm{DOTA}-\mathrm{ZOL}$ treatment. Though the difference in the haemoglobin levels post-second cycle was significant $(P<0.0001)$, only two patients in the series experienced grade II anaemia after the $\left[{ }^{177} \mathrm{Lu}\right] \mathrm{Lu}-\mathrm{DOTA}-\mathrm{ZOL}$ therapy; nadir was around 4 weeks and subsequently recovered (Fig. 6). None of the patients had shown renal toxicity and other side effects like hypercalcemia.
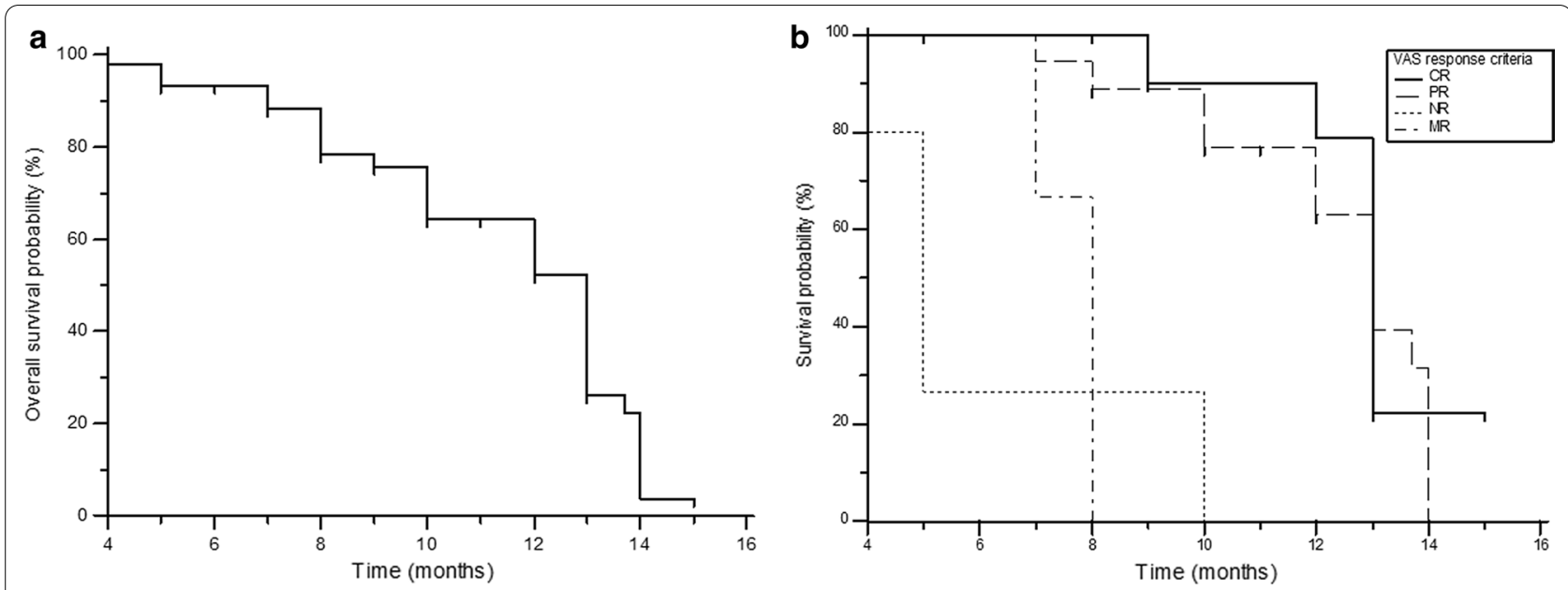

Fig. 4 a Kaplan-Meier analysis: overall survival. b Overall survival, according to the response category as per VAS response criteria 


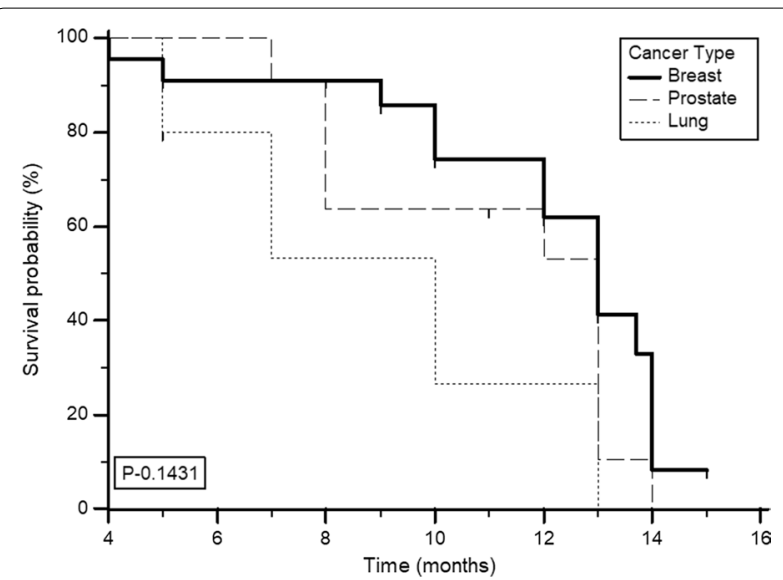

Fig. 5 Kaplan-Meier analysis: overall survival according to the type of cancer

\section{Discussion}

Three independent randomised, placebo-controlled trials on zoledronic acid in about 3000 patients demonstrating hormone-resistant metastatic prostate cancer [33], breast cancer [34], have demonstrated a reduction in skeletalrelated events (SREs) and proved it clinically effective. Subsequently, zoledronate received regulatory approval for the prevention of SREs and treating bone metastases. The labelling of zoledronate to beta- and alpha-emitting radionuclides like ${ }^{177} \mathrm{Lu}$ and ${ }^{225} \mathrm{Ac}$, respectively, was a logical development $[19,20]$.

Though EDTMP has been proved effective for bone pain palliation $[8],\left[{ }^{177} \mathrm{Lu}\right] \mathrm{Lu}-\mathrm{DOTA}-\mathrm{ZOL}$ proves superior to the former agent in certain aspects. In comparison with EDTMP, zoledronate demonstrates higher osteoclastic bone affinity and excellent anti-resorptive

Table 6 Laboratory parameters at baseline and post-[ $\left.{ }^{177} \mathrm{Lu}\right] \mathrm{Lu}-\mathrm{DOTA}-\mathrm{ZOL}$ treatment

\begin{tabular}{|c|c|c|c|c|c|c|}
\hline \multirow[t]{2}{*}{ Parameters } & \multicolumn{3}{|c|}{ Patients receiving single cycle } & \multicolumn{3}{|l|}{ Patients receiving 2 cycles } \\
\hline & Baseline (median, IQR) & $\begin{array}{l}\text { Post-treatment (median, } \\
\text { IQR) }\end{array}$ & $P$ value & Baseline (median, IQR) & $\begin{array}{l}\text { Post-treatment } \\
\text { (median, IQR) }\end{array}$ & $P$ value \\
\hline Haemoglobin ( $\mathrm{g} / \mathrm{dL})$ & $10.2(9.6-10.6)$ & $10(8.9-11)$ & 0.129 & $14.7(10-12.4)$ & $13(9.2-11)$ & $<0.0001$ \\
\hline Platelets (lakhs/uL) & $172(140.2-198)$ & $143(129.2-198.5)$ & 0.039 & $223(164-284.25)$ & $217(170-275.5)$ & 0.421 \\
\hline Leukocytes $10^{9} / \mathrm{L}$ & $6760(4210-11,800)$ & $5400(3700-10,800$ & 0.291 & $6700(4280-12,000)$ & $5400(3800-10,900)$ & 0.298 \\
\hline Creatinine (mg/dL) & $0.8(0.63-0.96)$ & $0.79(0.6-0.94)$ & 0.259 & $0.7(0.7-0.9)$ & $0.8(0.7-0.8)$ & 0.174 \\
\hline ALP (IU/L) & $282(190-480)$ & $189(140-280)$ & 0.0001 & $278(186-390)$ & $230(142-340)$ & 0.003 \\
\hline
\end{tabular}

$A L P$ alkaline phosphatase, IQR interquartile range

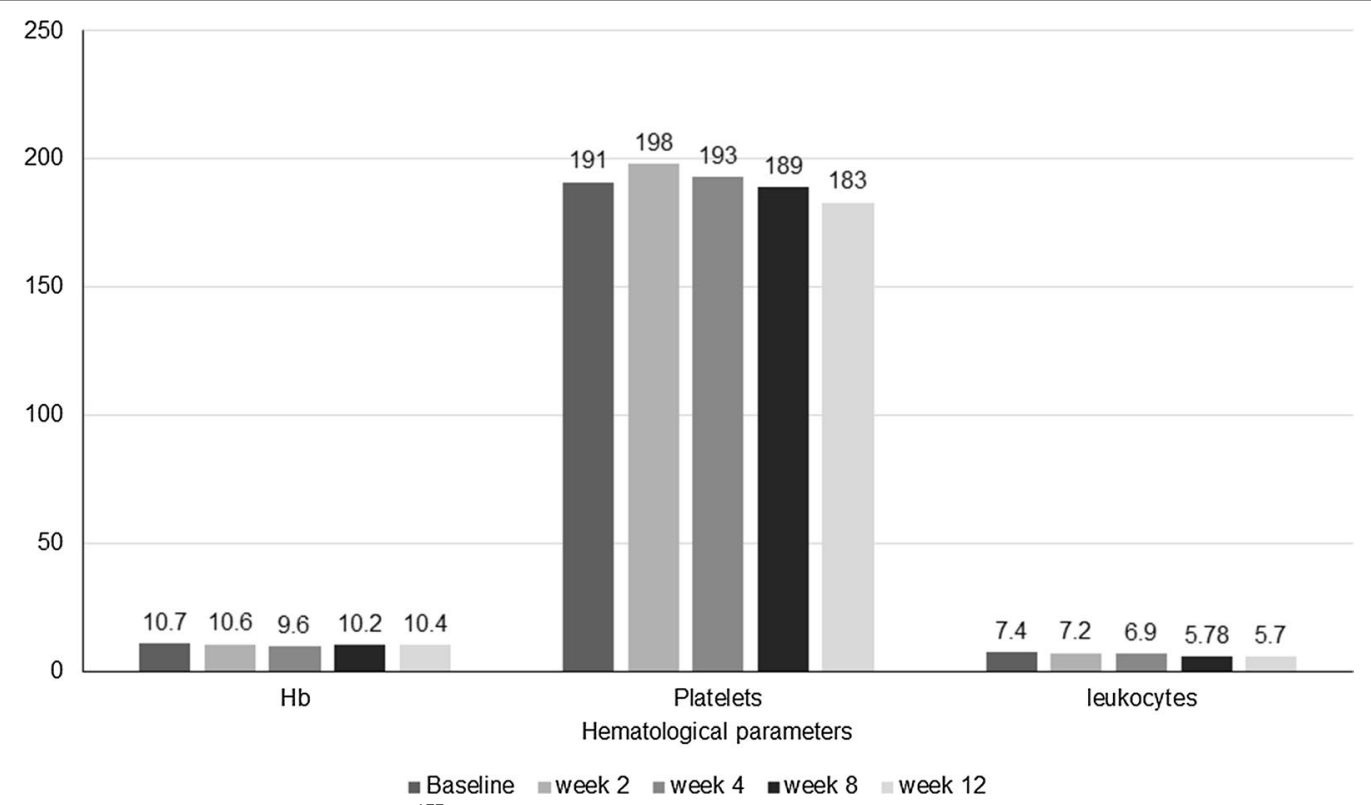

Fig. 6 Median haematological parameters after [ $\left.{ }^{177} \mathrm{Lu}\right] \mathrm{Lu}-\mathrm{DOTA}-\mathrm{ZOL}$ therapy 
properties. While both the agents are non-bio-transformative, rapid clearance of $\left[{ }^{177} \mathrm{Lu}\right] \mathrm{Lu}-\mathrm{DOTA}-\mathrm{ZOL}$ from blood, in contrast to [ $\left.{ }^{177} \mathrm{Lu}\right] \mathrm{Lu}$-EDTMP, dosimetric studies report a higher absorbed dose to the trabecular bone surface (12.17 vs. $10.02 \mathrm{mSv} / \mathrm{MBq}$ ), cortical bone surface $(9.524 \pm 0.803$ vs. $7.839 \pm 0.655)$, and a noticeably lesser muscle uptake [27]. Therefore, $\left[{ }^{177} \mathrm{Lu}\right] \mathrm{Lu}-\mathrm{DOTA}-\mathrm{ZOL}$ theoretically would have a better treatment efficacy. Moreover, unlike EDTMP, DOTA-ZOL complexes with ${ }^{68} \mathrm{Ga}$ and ${ }^{177} \mathrm{Lu} /{ }^{225} \mathrm{Ac}$ facilitate [19] a theranostic pair for practicing precision oncology.

However, to date, there is no study published in the literature showing therapeutic efficacy, safety, and toxicity of $\left[{ }^{177} \mathrm{Lu}\right] \mathrm{Lu}-\mathrm{DOTA}-\mathrm{ZOL}$ in bone pain palliation in metastatic cancer patients. Hence, the current research focuses on the efficacy and safety study of $\left[{ }^{177} \mathrm{Lu}\right]$ Lu-DOTA-ZOL pain palliation therapy in patients with bone metastases.

Well, in line with the literature [1], 85\% of the patients treated comprised of skeletal metastases from breast and prostate cancer. Interestingly, a significant component of our patients $(70 \%)$ managed had an ECOG performance state of 3 or 4 , and $35 \%$ of patients presented with either diffuse or super-scan reflecting the high tumour burden and poor ECOG status, which is a real-life scenario at the clinics. These sets of patients were refractory to the ongoing therapy options, including strong analgesics.

Khawar et al. [21] reported an estimated maximum tolerable dose (MTD) of $\left.{ }^{[177} \mathrm{Lu}\right] \mathrm{Lu}-\mathrm{DOTA}-\mathrm{ZOL}$ to be 3.6-5.0 GBq can be administered based on the 2 Gy bone marrow limit. In the present study, we administered a mean cumulative dose of $2.1 \pm 0.6 \mathrm{GBq}$ and ranged 1.3-2.7 GBq over a median of 2 cycles at three-monthly intervals; these administered activities were well within the prescribed limits. According to Khawar et al. [21], if the administered activities do not exceed MTD, the threshold adsorbed doses for the critical organ, namely kidneys, shall be within the predicted limit. Patients who responded from the first cycle of $\left[{ }^{177} \mathrm{Lu}\right] \mathrm{Lu}$-DOTA-ZOL were counselled or self-consented for the second cycle, while patients who were not willing to undergo the next cycle of treatment or those who did not experience any relief in pain after the first cycle of $\left[{ }^{177} \mathrm{Lu}\right] \mathrm{Lu}$-DOTAZOL were not treated further. Another interesting find observed was that those patients who experienced minimal response from the 1st cycle of $\left[{ }^{177} \mathrm{Lu}\right] \mathrm{Lu}-\mathrm{DOTA}-\mathrm{ZOL}$ treatment when re-challenged with the 2nd cycle did not encounter further reduction in the pain.

The current study reports an ORR of $90 \%$, which is well within the range of response rate with other bone-seeking pain palliating agent $\left[{ }^{177} \mathrm{Lu}\right] \mathrm{Lu}$-EDTMP ranging between 83 and $86 \%[8,9]$. Interestingly, pain relief was initiated within $\leq 7$ days after the $\left[{ }^{177} \mathrm{Lu}\right]$
Lu-DOTA-ZOL treatment and lasted up to 10 months. In a previous study using $\left[{ }^{177} \mathrm{Lu}\right] \mathrm{Lu}$-EDTMP, we had reported response durations that varied from 2 weeks to 4 months from the onset of pain relief [8]. Detailed analysis revealed a similar ORR of $82 \%$ in prostate cancer patients when compared to the historic $\left[{ }^{177} \mathrm{Lu}\right]$ Lu-EDTMP study (ORR 84\%) [8]. However, a remarkably high ORR of $100 \%$ was observed in the breast cancer cohort of the present study in contrast to $92 \%$ in the historic $\left[{ }^{177} \mathrm{Lu}\right] \mathrm{Lu}-\mathrm{EDTMP}$ study [8]. Though data regarding the response pattern in bone metastases from lung cancer are limited, Ye et al. [35] reported a treatment efficacy rate of $75.4 \%$ in the lung cancer group, but with $2.22 \mathrm{MBq} / \mathrm{kg}{ }^{89} \mathrm{SrCl}_{2}$. In agreement with our results, they reported efficacy was lower in patients with bone metastases from the lung cancer sub-group than in those with bone metastasis from breast and prostate cancer [35].

The 1 -year survival rate was $55.4 \%$ and was significantly higher when compared to that of the $\left[{ }^{177} \mathrm{Lu}\right] \mathrm{Lu}$-EDTMP historical data set $[35 \%$ and $38 \%$ in the two-level dose group] [8], but drastically dropped to $26 \%$ at 13 months. This drop of overall survival is not clearly understood; however, it could be attributed to the aggressive biology of the tumour and the extent of spread. Among the cancer types, similar overall survival was noted in patients with breast and prostate cancer (13 months) and a slightly decreased survival in bone metastases patients with primary lung cancer. Attributed to the superior radiobiological properties of the alpha emitter, radium-223 chloride, Parker et al. [4] in their RCT, involving 921 patients with bone metastases from prostate cancer, outlined an OS of 14.9 months. Nilsson et al. [36] performed a survival follow-up of the phase-II RCT in which bone metastases patients from prostate cancer were treated with radium-223 chloride, and their study findings suggested a survival benefit of 65 weeks versus 46 weeks in patients treated with a single dose of radium-223 chloride and placebo group, respectively.

Analgesic consumption was recorded according to the analgesic scoring of EORTC protocol. Among our recruited patients, 15 patients were on opioid analgesic morphine before radionuclide therapy out of whom 12 responded to $\left[{ }^{177} \mathrm{Lu}\right] \mathrm{Lu}-\mathrm{DOTA}-\mathrm{ZOL}$ treatment and thus were weaned off morphine. It is these set of patients whom the pain palliation was most beneficial as they have exhausted the available pain relief options. Interestingly, reassuring results were obtained with $\left[{ }^{177} \mathrm{Lu}\right]$ Lu-DOTA-ZOL in patients demonstrating extensive skeletal metastases and those presenting with superscan or diffuse involvement of the bone. The relief in pain was also reflected in their KPS and ECOG performance status. Toxicity related to $\left[{ }^{177} \mathrm{Lu}\right] \mathrm{Lu}-\mathrm{DOTA}-\mathrm{ZOL}$ 
therapy was minimal, and no grade III/IV toxicities were documents.

\section{Limitations}

The significant limitations of this cohort study are the non-randomised study, and no parallel arm was used. Although the results of $\left[{ }^{177} \mathrm{Lu}\right] \mathrm{Lu}-\mathrm{DOTA}-\mathrm{ZOL}$ were comparable to historical data of $\left[{ }^{177} \mathrm{Lu}\right] \mathrm{Lu}$-EDTMP, a randomised control trial to compare the efficacy and safety of two bone-seeking palliative agents is urgently desired to take this agent further.

\section{Conclusion}

$\left[{ }^{177} \mathrm{Lu}\right] \mathrm{Lu}-\mathrm{DOTA}-\mathrm{ZOL}$ is safe, effective, and an ideal agent in the treatment of metastatic bone pain. Thanks to its characteristics as theranostics, it allows for patientindividual therapies and perfectly matches the expectations for precision oncology. Interestingly, $\left[{ }^{177} \mathrm{Lu}\right]$ Lu-DOTA-ZOL is not only effective in addressing bone metastases derived from breast cancer; it shows clear evidence also for prostate and lung cancers. $\left[{ }^{177} \mathrm{Lu}\right]$ Lu-DOTA-ZOL pain palliation treatment demonstrated an ORR of $90 \%$ with $27.5 \%$ of complete response and $50 \%$ of partial response.

\begin{abstract}
Abbreviations
IQR: Interquartile range; VAS: Visual analogue score; AS: Analgesic score; ECOG: Eastern Cooperative Oncology Group Assessment performance status; KPS: Karnofsky performance status; SREs: Skeletal-related events; EBRT: External beam radiotherapy; EDTMP: Ethylene diamine tetramethylene phosphonic acid; BPAMD: 4-\{[(Bis(phosphonomethyl)) carbamoyl]methyl\}-7,10-bi (carboxymethyl)-1,4,7,10-tetraazacyclododec-1-YI) acetic acid; DOTA-ZOL: Zoledronate; BSA: Body surface area; EORTC: European Organization of Research and Treatment of Cancer; $\mathrm{LuCl}_{3}$ : Lutetium chloride; GFR: Glomerular filtration rate; $\mathrm{CBC}$ : Complete blood counts; KFT: Kidney function tests; ALP: Alkaline phosphatase; CR: Complete remission; PR: Partial remission; MR: Minimal response; NR: No response; PET/CT: Positron emission tomography/ computed tomography; NCI-CTC: National Cancer Institute's Common Toxicity Criteria; OS: Overall survival; SD: Standard deviation; MTD: Maximum tolerable dose; ORR: Overall response rate.
\end{abstract}

\section{Acknowledgements}

We would like to acknowledge Department of Nuclear Chemistry, Johannes Gutenberg University, Mainz, Germany, for providing the zoledronate (DOTAZOL) molecule which was used in this study.

\section{Author contributions}

MPY and SB helped in patient treatment, data collection, and manuscript writing. MM contributed to radiochemistry; synthesis of DOTA ZOL; and standardisation of radiolabelling. FR helped in radiochemistry; synthesis of DOTA ZOL; standardisation of radiolabelling; and manuscript review. CB planned the study, patient recruitment, patient treatment, follow-up, image assessment, and manuscript writing and final review. All authors read and approved the final manuscript.

\section{Funding}

No funding was received from any organisation to conduct this study.

Availability of data and materials

Data and material are available.
Ethical approval and consent to participate

Ref. No IEC/349/4/2020. Name of ethics committee is Institute Ethics committee, AllMS. A written informed consent was obtained from all the patients before commencing the treatment.

\section{Consent for publication}

All the authors gave their consent for the publication of this article.

Competing interests

The authors declare that they have no competing interests.

\section{Author details}

1 Department of Nuclear Medicine, Room No: 59-A, Thyroid Clinic, All India Institute of Medical Sciences (AllMS), Ansari Nagar, New Delhi 110029, India. ${ }^{2}$ Department of Nuclear Chemistry, Johannes Gutenberg University, Fritz-Strassmann-Weg 2, 55126 Mainz, Germany.

Received: 16 July 2020 Accepted: 21 September 2020

Published online: 28 October 2020

\section{References}

1. Roodman GD. Mechanisms of bone lesions in multiple myeloma and lymphoma. Cancer. 1997;80:1557-63.

2. WHO guidelines for the pharmacological and radiotherapeutic management of cancer pain in adults and adolescents. Geneva: World Health Organization; 2018.

3. Tong D, Gillick L, Hendrickson FR. The palliation of symptomatic osseous metastases: final results of the study by the Radiation Therapy Oncology Group. Cancer. 1982;50:893-9.n

4. Parker C, Nilsson S, Heinrich D, Helle SI, O'Sullivan JM, Fosså SD, et al. Alpha emitter radium-223 and survival in metastatic prostate cancer. N Engl J Med. 2013;369:213-23.nn

5. Pillai MR, Chakraborty ST, Venkatesh M, Ramamoorthy N. Production logistics of ${ }^{177}$ Lu for radionuclide therapy. Appl Radiat Isot. 2003;59:10918.nn

6. Lebedev NA, Novgorodov AF, Misiak R, Brockmann J, Rösch F. Radiochemical separation of no-carrier-added ${ }^{177} \mathrm{Lu}$ as produced via the ${ }^{176} \mathrm{Yb}(\mathrm{n}$, g) ${ }^{177} \mathrm{Yb}^{1}{ }^{177 L} \mathrm{u}$ process. Appl Radiat Isot. 2000;53:421-5.n

7. Mazzarri S, Guidoccio F, Mariani G. The emerging potential of ${ }^{177} \mathrm{Lu}-$ EDTMP: an attractive novel option for radiometabolic therapy of skeletal metastases. Clin Transl Imaging. 2015;3:167-8.n

8. Agarwal KK, Singla S, Arora G, Bal C. . ${ }^{17}$ Lu-EDTMP for palliation of pain from bone metastases in patients with prostate and breast cancer: a phase II study. Eur J Nucl Med Mol Imaging. 2015;42:79-88.n

9. Alavi M, Omidvari S, Jalilian A, Mehdizadeh A, Bahrami-Samani A. Metastatic bone pain palliation using ${ }^{177} \mathrm{Lu}$-ethylenediamine tetramethylene phosphonic acids. World J Nucl Med. 2015;14:109-15.nn

10. Rösch F, Baum RP. Generator-based PET radiopharmaceuticals for molecular imaging of tumors: on the way to THERANOSTICS. Dalton Trans. 2011:40:6104-11.n

11. Fellner M, Baum RP, Peters JA, Lukeš I, Hermann P, Prasad V, et al. PET/CT imaging of osteoblastic bone metastases with ${ }^{68} \mathrm{Ga}$-bisphosphonatesfirst in the human study. Eur J Nucl Med Mol Imaging. 2010;37:834.nn

12. Fellner M, Riss P, Loktionova NS, Zhernosekov KP, Thews O, Geraldes CFGC, et al. Comparison of different phosphorus-containing ligands complexing 68Ga for PET-imaging of bone metabolism. Radiochim Acta. 2011;99:43-51.n

13. Fellner M, Bisalski B, Bausbacher N, Kubícek V, Hermann P, Rösch F, et al. ${ }^{6} 8 \mathrm{Ga}$-BPAMD: PET-imaging of bone metastases with a generator based positron emitter. Nucl Med Biol. 2012;39:993-9.nn

14. Meckel M, Fellner M, Thieme N, Bergmann R, Kubicek V, Rösch F. In vivo comparison of DOTA based 68Ga-labelled bisphosphonates for bone imaging in non-tumor models. Nucl Med Biol. 2013;40:823-30.n

15. Meckel M, Nauth A, Timpe J, Zhernosekov K, Puranik AD, Baum RP, et al. Development of a [ $\left.{ }^{177} \mathrm{Lu}\right] \mathrm{BPAMD}$ labeling kit and an automated synthesis module for routine bone targeted endoradiotherapy. Cancer Biother Radiopharm. 2015;30:94-9.n 
16. Bergmann R, Meckel M, Kubíček V, Pietzsch J, Steinbach J, Hermann P, et al. ${ }^{177}$ Lu-labeled macrocyclic bisphosphonates for targeting bone metastasis in cancer treatment. EJNMMI Res. 2016;6:5.n

17. Meckel M, Kubíček V, Hermann P, Miederer M, Rösch F. A DOTA based bisphosphonate with an albumin binding moiety for delayed body clearance for bone targeting. Nucl Med Biol. 2016;43:670-8.nn

18. Meckel M, Bergmann R, Miederer M, Roesch F. Bone targeting compounds for radiotherapy and imaging: *Me(III)-DOTA conjugates of bisphosphonic acid, pamidronic acid, and zoledronic acid. EJNMMI Radiopharm Chem. 2017;1:14.nn

19. Pfannkuchen N, Meckel M, Bergmann R, Bachmann M, Bal C, Sathekge M, et al. Novel radiolabeled bisphosphonates for PET diagnosis and endoradiotherapy of bone metastases. Pharmaceuticals (Basel). 2017;10:E45.n

20. Pfannkuchen N, Bausbacher N, Pektor S, Miederer M, Rösch F. In vivo evaluation of $\left[{ }^{225} \mathrm{Ac}\right] \mathrm{Ac}$-DOTAZOL for a-therapy of bone metastases. Curr Radiopharm. 2018;11:223-30.nn

21. Khawar A, Eppard E, Roesch F, Ahmadzadehfar H, Kürpig S, Meisenheimer $M$, et al. Biodistribution and post-therapy dosimetric analysis of $\left[{ }^{177} \mathrm{Lu}\right] \mathrm{Lu}$ DOTAZOL in patients with osteoblastic metastases: first results. EJNMMI Res. 2019a;9:102.nn

22. Khawar A, Eppard E, Roesch F, Ahmadzadehfar H, Kürpig S, Meisenheimer $M$, et al. Preliminary results of biodistribution and dosimetric analysis of $\left[{ }^{68} \mathrm{Ga}\right] \mathrm{Ga}$-DOTA-ZOL: a new zoledronate-based bisphosphonate for PET/ CT diagnosis of bone diseases. Ann Nucl Med. 2019b;6(404-13):7.n

23. Chakraborty S, Das T, Sarma HD, Venkatesh M, Banerjee S. Comparative studies of ${ }^{177}$ Lu-EDTMP and ${ }^{177}$ Lu-DOTMP as potential agents for palliative radiotherapy of bone metastasis. Appl Radiat Isot. 2008;66:1196-205.n

24. Das T, Shinto A, Kamaleshwaran KK, Banerjee S. Theranostic treatment of metastatic bone pain with ${ }^{177}$ Lu-DOTMP. Clin Nucl Med. 2016;41:966-7.n

25. Russell RGG. Bisphosphonates: mode of action and pharmacology. Pediatrics. 2007;119(Suppl 2):150-62.nn

26. Nikzad M, Jalilian AR, Shirvani-Arani S, Bahrami-Samani A, Golchoubian $\mathrm{H}$. Production, quality control and pharmacokinetic studies of ${ }^{177} \mathrm{Lu}$ zoledronate for bone pain palliation therapy. J Radioanal Nucl Chem. 2013:298:1273-81.nnn

27. Yousefnia H, Zolghadri S, Jalilian AR. Absorbed dose assessment of ${ }^{177} \mathrm{Lu}-$ zoledronate and ${ }^{177}$ Lu-EDTMP for human based on biodistribution data in rats. J Med Phys. 2015;40:102-8.n
28. Common Terminology Criteria for Adverse Events (CTCAE) v5.0 Publish Date: November 27, 2017.

29. McCaffery M, Pasero C. Pain: clinical manual. 2nd ed. St Louis: Mosby; 1999.n

30. Schag CC, Heinrich RL, Ganz PA. Karnofsky performance status revisited: reliability, validity, and guidelines. J Clin Oncol. 1984;2:187-93.nn

31. Crooks V, Waller S, Smith T, Hahn TJ. The use of the Karnofsky Performance Scale in determining outcomes and risk in geriatric outpatients. J Gerontol. 1991:46:M139-44.n

32. Thapa P, Nikam D, Das T, Sonawane G, Agarwal JP, Basu S. Clinical efficacy and safety comparison of ${ }^{177}$ Lu-EDTMP with ${ }^{153} \mathrm{Sm}$-EDTMP on an equidose basis in patients with painful skeletal metastases. J Nucl Med. 2015:56:1513-9.n

33. Saad F, Gleason DM, Murray R, Tchekmedyian S, Venner P, Lacombe L, et al. Zoledronic Acid Prostate Cancer Study Group. A randomized, placebo-controlled trial of zoledronic acid in patients with hormone-refractory metastatic prostate carcinoma. J Natl Cancer Inst. 2002;94:1458-68. $\mathrm{nn}$

34. Rosen LS, Gordon D, Kaminski M, Howell A, Belch A, Mackey J, et al. Zoledronic acid versus pamidronate in the treatment of skeletal metastases in patients with breast cancer or osteolytic lesions of multiple myeloma: a phase III, double-blind, comparative trial. Cancer J. 2001;7:377-87.nn

35. Ye X, Sun D, Lou C. Comparison of the efficacy of strontium-89 chloride in treating bone metastasis of lung, breast, and prostate cancers. J Cancer Res Ther. 2018;14:S36-40.n

36. Nilsson S, Franzén L, Parker C, et al. Two-year survival follow-up of the randomized, double-blind, placebo-controlled phase II study of radium-223 chloride in patients with castration-resistant prostate cancer and bone metastases. Clin Genitourin Cancer. 2013;11(1):20-6. https:// doi.org/10.1016/j.clgc.2012.07.002.nn

\section{Publisher's Note}

Springer Nature remains neutral with regard to jurisdictional claims in published maps and institutional affiliations.

\section{Submit your manuscript to a SpringerOpen ${ }^{\circ}$ journal and benefit from:}

- Convenient online submission

- Rigorous peer review

- Open access: articles freely available online

- High visibility within the field

Retaining the copyright to your article

Submit your next manuscript at $\boldsymbol{\nabla}$ springeropen.com 